\title{
TOPOLOGICAL CLASSIFICATION OF IRREDUCIBLE PLANE CURVE SINGULARITIES IN TERMS OF WEIERSTRASS POLYNOMIALS
}

\author{
CHUNGHYUK KANG
}

(Communicated by Peter Li)

\begin{abstract}
Let $f(z, y)$ be analytically irreducible at 0 and $f(0)=0$. Then the plane curve singularity defined by $f$ has the same topological type as the curve defined by $f_{k+1}$ for some $k \geq 0$ where $f_{1}=z^{a}+y^{b}, f_{2}=f_{1} n_{21}+$ $y^{m_{11}} z^{m_{12}}, f_{3}=f_{2}^{n_{31}}+f_{1}^{n_{22}} y^{m_{21}} z^{m_{22}}, \ldots$ are defined by induction on $k$ with distinct numerical conditions topologically invariant. Moreover, we give an easy alternate proof of Zariski's topological classification theorem of irreducible plane curve singularities.
\end{abstract}

\section{INTRODUCTION}

Let $\mathbb{C}\{y, z\}$ or $\mathscr{O}_{0}$ be the ring of convergent power series at $(y, z)=(0,0)$. Suppose that $f$ is irreducible in $\mathscr{O}_{0}$ with $f(0)=0$. Now consider the plane curve singularity defined by $f$. In decreasing order in the degree of $z, f$ can be written as follows: $f=a_{0} z^{n}+a_{1} y^{\alpha_{1}} z^{n-1}+\cdots+a_{n} y^{\alpha_{n}}$ where $a_{0}, a_{n}$ are units in $\mathscr{O}_{0}$, and for $1 \leq i \leq n-1$ each $a_{i}$ is a unit in $\mathscr{O}_{0}$ if it exists, and the $\alpha_{i}$ are positive integers. Then by Theorem 2.2 we easily prove that if $f$ is irreducible in $\mathscr{O}_{0}, \frac{\alpha_{n}}{n} \leq \frac{\alpha_{i}}{i}$ for all $i$. Also, if $\left(n, \alpha_{n}\right)=1$, we show that the above inequality becomes sufficient for the irreducibility of $f$ in $\mathscr{O}_{0}$. Moreover, even if $\left(n, \alpha_{n}\right)>1$, the necessary condition may be said to be sufficient in an inductive sense in terms of blow-ups. Using this fact we can classify topologically irreducible plane curve singularities in terms of Weierstrass polynomials (Theorem 4.2). Finally, in a very elementary way we are going to prove Zariski's topological classification theorem of irreducible plane curve singularities in terms of parametrization (Theorem 4.4).

\section{KNOWN PRELIMINARIES}

Let $V=\{(y, z): f(y, z)=0\}$ be an analytic subvariety of a polydisc in $\mathbb{C}^{2}$ with $(0,0) \in V$, and $(0,0)$ a singular point where $f$ is holomorphic near $(0,0)$ and square-free. Let $\pi: M \rightarrow \mathbb{C}^{2}$ be a blow-up of $\mathbb{C}^{2}$ at $(0,0)$. Let $(u, v)$ and $\left(u^{\prime}, v^{\prime}\right)$ be coordinate patches for $M$ with $\pi(u, v)=(y, z)=$

Received by the editors July $15,1992$.

1991 Mathematics Subject Classification. Primary 32S15, 14E15.

Supported in part by the Korean Ministry of Education and GARC at Seoul National University, 1992. 
$(u v, v)$ and $\pi\left(u^{\prime}, v^{\prime}\right)=(y, z)=\left(v^{\prime}, u^{\prime} v^{\prime}\right)$. Let $e$ be the multiplicity of $f$ at $(0,0) ; e \geq 2$. Then $\pi^{-1}(V)$, the total transform of $V$, is given locally by $f(u v, v)=v^{e} f_{1}(u, v)$ and $f\left(v^{\prime}, u^{\prime} v^{\prime}\right)=v^{e} f_{2}\left(u^{\prime}, v^{\prime}\right)$. Then we call $V^{(1)}$ the proper transform of $V$ at $(0,0)$. Note that if $f$ is irreducible in $\mathscr{O}_{0}$ then just one coordinate patch is needed for the study of $V^{(1)}$. After $m$ iterations of blow-ups, let $\tau_{m}=\pi \circ \pi_{2} \circ \ldots \circ \pi_{m}: M^{(m)} \rightarrow \mathbb{C}^{2}$. Let $V^{(m)}$ be the proper transform of $V$ under $\tau_{m}$. Let $E^{(m)}=\tau_{m}^{-1}(0,0)$. Then $E^{(m)}$ is, by definition, an exceptional set of the first kind. Let $E^{(m)}=\bigcup E_{i}, 1 \leq i \leq m$, be the decomposition of $E^{(m)}$ into irreducible components. Each $E_{i}$ is called an exceptional curve of the first kind. Let $\left(f \circ \tau_{m}\right)=V^{(m)}+\sum e_{i} E_{i}, 1 \leq i \leq m$, be the divisor of $f \circ \tau_{m}$. Then we have the following well-known theorem.

Theorem 1.1. Let $V=\{(y, z): f(y, z)=0\}$ be an analytic subvariety of a polydisc in $\mathbb{C}^{2}$ with $(0,0) \in V$ and $(0,0)$ only a singular point where $f$ is in $\mathscr{O}_{0}$ and square-free. There exists an analytic manifold $M$ by finitely many $(m)$ blow-ups, $\tau_{m}: M \rightarrow \mathbb{C}^{2}$, such that if $R$ is the set of regular points on $V$ then $\tau_{m}: \overline{\tau_{m}^{-1}(R)} \rightarrow V$ is a resolution of the singular point $(0,0)$ of $V$, where $\overline{\tau_{m}^{-1}(R)}$ is the closure of $\tau_{m}^{-1}(R)$ in $M$.

Corollary 1.2. Under the same assumption of Theorem 1.1, after additional blow-ups any two components of $V^{(m)}$ and $\bigcup E_{i}, 1 \leq i \leq m$, meet with normal crossings whenever they meet and no three distinct components of $V^{(m)}$ and $\bigcup E_{i}$ meet, where $V^{(m)}$ and $\bigcup E_{i}$ are defined just before Theorem 1.1 .

Definition 1.3. Let $V=\{f(y, z)=0\}$ and $W=\{g(y, z)=0\}$ be analytic subvarieties of a polydisc in $\mathbb{C}^{2}$ with $(0,0) \in V$ and $(0,0) \in W$, where $V$ and $W$ have the only singular point at the origin, and $f, g$ are in $\mathscr{O}_{0}$ and square-free. $V$ and $W$ are said to have a homeomorphic resolution (or topological or equisingular) if, using the composition of the same number of successive blow-ups $\tau_{m}: M \rightarrow \mathbb{C}^{2},\left(f \circ \tau_{m}\right)$ and $\left(g \circ \tau_{m}\right)$ are equivalent as divisors in the sense of Corollary 1.2. Then denote this relation by $f \sim g$.

\section{A CRITERION OF IRREDUCIBILITY OF PLANE CURVES}

Lemma 2.1 (Hensel's Lemma). Let $f(y, z)=a_{0} z^{n}+a_{1} y^{\alpha_{1}} z^{n-1}+\cdots+a_{n} y^{\alpha_{n}}$ be irreducible in $\mathscr{O}_{0}$, where each $a_{i}$ is a unit in $\mathscr{O}_{0}$ if it exists, and the $\alpha_{i}$ are positive integers. Let $m$ be the multiplicity of $f$ at 0 . Then $m=n$ or $\alpha_{n}$. If $n=\alpha_{i}+n-i$ for some $i$, then $n=\alpha_{i}+n-i$ for all $i=1, \ldots, n$, and so $f$ can be written in a power series as follows: $f=f_{n}(y, z)+$ terms of degree $>n$, where $f_{n}$ is a homogeneous polynomial of degree $n$ with $f_{n}=(a y+b z)^{n}$ for some $a, b \in \mathbb{C}$.

Theorem 2.2. Let $f(y, z)=a_{0} z^{n}+a_{1} y^{\alpha_{1}} z^{n-1}+\cdots+a_{n} y^{\alpha_{n}}$ be irreducible in $\mathscr{O}_{0}$, where each $a_{i}$ is a unit in $\mathscr{O}_{0}$ if it exists, and the $\alpha_{i}$ are positive integers. Then $\frac{\alpha_{n}}{n} \leq \frac{\alpha_{i}}{i}$ for all $i$. Moreover, if $\alpha_{n}=n k$ for some integer $k$, then $\frac{\alpha_{n}}{n}=\frac{\alpha_{i}}{i}$ for all $i=1, \ldots, n-1$.

Proof. We are going to prove the above assertion by induction on the multiplicity of $f$. Without loss of generality we may assume that $\alpha_{n} \leq n$ for the following reason: If $k n<\alpha_{n} \leq(k+1) n$ for some positive integer $k$, then use $k$ blow-ups and Lemma 2.1 because blow-ups preserve irreducibility 
of $f$ in $\mathscr{O}_{0}$ and $\frac{a_{n}}{n} \leq \frac{\alpha_{i}}{i}$ in $f$ if and only if $\frac{\alpha_{n}-k n}{n} \leq \frac{\alpha_{i}-k i}{i}$ in $f_{k}$, where $f\left(v, u v^{k}\right)=v^{k n} f_{k}$ and $f_{k}=a_{0} v^{n}+\cdots+a_{i} u^{\alpha_{i}-k i} v^{n-i}+\cdots+a_{n} u^{\alpha_{n}-k n}$. If $n=\alpha_{n}$, then by Lemma $2.1 n=a_{i}+n-i$ for all $i$, and so $\frac{\alpha_{n}}{n}=\frac{\alpha_{i}}{i}=1$. Let $n>\alpha_{n}$. Then there is a positive integer $r$ such that $0<n-r \alpha_{n} \leq \alpha_{n}$. Repeat $r$ blow-ups at $(y, z)=(0,0)$. Then the local defining equation for the $r$ th total transform may be given by $f(y, z)=f\left(u_{1} v_{1}^{r}, v_{1}\right)=v_{1}^{\alpha_{n}} f_{r}$, where $f_{r}=a_{0} v_{1}^{n-r \alpha_{n}}+\cdots+a_{i} u_{1}^{\alpha_{i}} v_{1}^{r \alpha_{i}+n-i-r \alpha_{n}}+\cdots+a_{n} u_{1}^{\alpha_{n}}$. Note that $n-r \alpha_{n}>0$ and $r \alpha_{i}+n-i-r \alpha_{n}>0$ by Lemma 2.1. If $n-r \alpha_{n}=\alpha_{n}$, then by Lemma 2.1 $\alpha_{i}+r \alpha_{i}+n-i-r \alpha_{n} \geq n-r$ for all $i$, i.e., $\alpha_{i}(r+1) \geq i$. Thus $\frac{\alpha_{i}}{i} \geq \frac{1}{r+1}=\frac{\alpha_{n}}{n}$ for all $i$. Let $n-r \alpha_{n}<\alpha_{n}$. Then we may assume that $r \alpha_{i}+n-i-r \alpha_{n}<n-r \alpha_{n}$. If $r \alpha_{i}+n-i-r \alpha_{n} \geq n-r \alpha_{n}$ for some $i$, then $n-r \alpha_{n}>0$ implies also that $\frac{\alpha_{n}}{n}<\frac{1}{r} \leq \frac{\alpha_{i}}{i}$. Also if $r \alpha_{i}+n-i-r \alpha_{n}=r \alpha_{j}+n-j-r \alpha_{n}$ for $i<j$, then $\alpha_{j}>\alpha_{i}$. So rewriting $f_{r}$ in decreasing order of $v$, by the induction assumption we get

$$
\frac{\alpha_{n}}{n-r \alpha_{n}} \leq \frac{\alpha_{i}}{n-r \alpha_{n}-\left(r \alpha_{i}+n-i-r \alpha_{n}\right)} \leq \frac{\alpha_{j}}{n-r \alpha_{n}-\left(r \alpha_{j}+n-j-r \alpha_{n}\right)} .
$$

Thus $\frac{\alpha_{n}}{n} \leq \frac{\alpha_{i}}{i} \leq \frac{\alpha_{j}}{j}$.

Corollary 2.3. Assume that the hypotheses of Theorem 2.2 are satisfied. If $\left(n, \alpha_{n}\right)=1$, then $f$ is irreducible in $\mathscr{O}_{0}$ if and only if $\frac{\alpha_{n}}{n}<\frac{\alpha_{i}}{i}$ for all $i \neq n$. Moreover, in this case $f \sim z^{n}+y^{\alpha_{n}}$ in $\mathscr{O}_{0}$.

Proof. Let $\left(n, \alpha_{n}\right)=1$. If $f$ is irreducible in $\mathscr{O}_{0}$, then $\frac{\alpha_{n}}{n}<\frac{\alpha_{i}}{i}$ by Theorem 2.2. To prove the converse, it suffices to follow the same induction argument as we have seen in the proof of Theorem 2.2.

\section{A CHARACTERIZATION OF IRREDUCIBLE PLANE CURVES}

Let $f(y, z)=a_{0} z^{n}+a_{1} y^{\alpha_{1}} z^{n-1}+\cdots+a_{n} y^{\alpha_{n}}$ be irreducible in $\mathscr{O}_{0}$, where each $a_{i}$ is a unit in $\mathscr{O}_{0}$ if it exists. By the Weierstrass Preparation Theorem and a nonsingular linear change of coordinate $f$ can be rewritten as follows:

$$
f=A\left(w^{n}+c_{2} y^{\gamma_{2}} w^{n-2}+\cdots+c_{n} y^{\gamma_{n}}\right),
$$

where $A$ is a unit in $\mathbb{C}\{y, w\}$ and the $c_{i}(y)$ are units in $\mathbb{C}\{y, w\}$ if they exist, and the $\gamma_{i}$ are positive integers. Then we need to find the relationship between $\alpha_{n}$ and $\gamma_{n}$. Note that $c_{1}$ is a zero.

Lemma 3.1. Under the above assumption, there are two cases.

(i) If $\alpha_{n}=n k$ for some integer $k$, then $\gamma_{n}>\alpha_{n}$ and $\left(\gamma_{n}, n\right)<n$.

(ii) If $\left(\alpha_{n}, n\right)<n$, then $\alpha_{n}=\gamma_{n}$.

Proof. The proof just follows from Lemma 2.1 or Theorem 2.2.

Lemma 3.2. Let $a$ and $b$ be positive integers with $(a, b)=1$. Then for any integer $n>a b-a-b$ there exist nonnegative integers $x$ and $y$ such that $n=a x+b y$. If $n>a b$ then $x$ and $y$ can be chosen both positive.

Proof. See [4].

Let $f=z^{n}+a_{2} y^{\alpha_{2}} z^{n-2}+\cdots+a_{n} y^{\alpha_{n}}$ be irreducible in $\mathscr{O}_{0}$ with multiplicity $n$, where the $a_{i}(y)$ are units for $2 \leq i \leq n$ in $\mathscr{O}_{0}$ if they exist. Note that $a_{1}=0$. If $\left(n, \alpha_{n}\right)=1$, by Corollary $2.3 f$ is irreducible if and only if $\frac{\alpha_{n}}{n}<\frac{\alpha_{i}}{i}$ for all 
$i \neq n$, and so $f \sim z^{n}+y^{\alpha_{n}}$ near $(0,0)$. Now assume that $d=\left(n, \alpha_{n}\right)>1$. By Lemma 3.1, $d<n$. Set $n=n_{1} d$ and $\alpha_{n}=k_{1} d$. Now consider $f$ as a convergent power series $\sum c_{\alpha \beta} y^{\alpha} z^{\beta}$. Look at each monomial in $f$ and rewrite $f$ in increasing order of $n_{1} \alpha+k_{1} \beta$ as follows:

$$
f=\sum_{0}+\sum_{1}+\sum_{2}+\cdots,
$$

where $\sum_{i}=\sum_{i} c_{\alpha \beta} y^{\alpha} z^{\beta}$ and each monomial of $\sum_{i}$ satisfies $n_{1} \alpha+k_{1} \beta=$ $n_{1} k_{1} d+m_{i}$ for each $i \geq 0$ and $0=m_{0}<m_{1}<m_{2}<\cdots$. After a finite number $h$ of blow-ups until the total order of the new exceptional curve $E_{h}=$ $\{r=0\}$ becomes $n_{1} k_{1} d$ in one of the local coordinates $(s, r)$, the local defining equation $f_{1}$ for the $h$ th proper transform of the curve defined by $f$ is given topologically as follows:

$$
f_{1}=(s+1)^{d}+c_{2} r^{e_{2}}(s+1)^{d-2}+\cdots+c_{d} r^{e_{d}},
$$

where each $c_{i}$ is a unit in $\mathbb{C}\{s+1, r\}$ if it exists, and the $e_{i}$ are positive integers. Note that $E_{h}$ is the first exceptional curve which has three distinct intersection points with two other exceptional curves and the proper transform. If $\left(d, e_{d}\right)=$ 1 , then $f_{1}$ is irreducible in $\mathbb{C}\{s+1, r\}$ if and only if $\frac{e_{i}}{i}>\frac{e_{d}}{d}$ for all $i \neq d$, and so $f_{1} \sim(s+1)^{d}+r^{e_{d}}$. Therefore, reversing blow-ups, we get by Lemma 3.2 that $f \sim\left(z^{n_{1}}+y^{k_{1}}\right)^{d}+y^{p} z^{q}$ in $\mathscr{O}_{0}$, where $\left(n_{1}, k_{1}\right)=1$, and $p n_{1}+q k_{1}=n_{1} k_{1} d+e_{d}$ with $\left(p n_{1}+q k_{1}, d\right)=1$. Observe that $q$ may be chosen with $q<n_{1} d$ since $n=n_{1} d$ is the multiplicity of $f$. If $g=\left(z^{n_{1}}+y^{k_{1}}\right)^{d}+y^{p_{1}} z^{q_{1}}$, then we can show that $g \sim f$ in $\mathscr{O}_{0}$ if and only if $p_{1} n_{1}+q_{1} k_{1}=p n_{1}+q k_{1}$. Let $\left(d, e_{d}\right)>1$. Note that $\left(d, e_{d}\right)$ may be equal to $e_{d}<d$. Put $d=n_{2} d_{2}$ and $e_{d}=k_{2} d_{2}$ with $d_{2}=\left(d, e_{d}\right)$. Write $f_{1}$ again in $\mathbb{C}\{s+1, r\}$. Then $f_{1}$ has the form

$$
f_{1}=\sum_{10}+\sum_{11}+\sum_{12}+\cdots,
$$

where $\sum_{1 i}=\sum b_{\alpha \beta}(s+1)^{\alpha} r^{\beta}$ and each term of $\sum_{1 i}$ satisfies $\alpha k_{2}+\beta n_{2}=$ $n_{2} k_{2} d_{2}+l_{i}$ for each $i \geq 0$ and $0=l_{0}<l_{1}<l_{2}<\ldots$. Then repeat those arguments just as above inductively. Thus the necessary condition of Theorem 2.2 for $f$ to be irreducible in $\mathscr{O}_{0}$ is sufficient in an inductive sense, as we have seen in the above discussion.

\section{A tOPOLOGICAL CLASSIFICATION \\ OF IRREDUCIBLE PLANE CURVE SINGULARITIES \\ IN TERMS OF WEIERSTRASS POLYNOMIALS}

Definition 4.1. Let

$$
f_{1}=z^{a}+y^{b}, \quad f_{2}=f_{1}^{n_{21}}+y^{m_{11}} z^{m_{12}}, \quad f_{3}=f_{2}^{n_{31}}+f_{1}^{n_{22}} y^{m_{21}} z^{m_{22}}, \ldots
$$

and

$$
f_{k+1}=f_{k}^{n_{k+1,1}}+f_{k-1}^{n_{k, 2}} f_{k-2}^{n_{k-1,3}} \cdots f_{1}^{n_{2, k}} y^{m_{k, 1}} z^{m_{k, 2}}
$$

be Weierstrass polynomials in $z$, where $2 \leq a<b, n_{i 1} \geq 2$ for $i=2, \ldots, k+$ 1 , and the multiplicity of $f_{j}$ is $a n_{21} n_{31} \cdots n_{j 1}$ for $j=2, \ldots, k+1$ at 0 . Define 
the new notations inductively as follows:

$$
\begin{aligned}
& \bar{f}_{2}\left(t_{1}, t_{2}\right)=a t_{1}+b t_{2}, \\
& \bar{f}_{3}\left(x_{2} ; t_{1}, t_{2}\right)=x_{2} \bar{f}_{2}\left(m_{11}, m_{12}\right)+n_{21} \bar{f}_{2}\left(t_{1}, t_{2}\right), \\
& \bar{f}_{4}\left(x_{3} ; x_{2}, t_{1}, t_{2}\right)=x_{3} \bar{f}_{3}\left(n_{22} ; m_{21}, m_{22}\right)+n_{31} \bar{f}_{3}\left(x_{2} ; t_{1}, t_{2}\right), \\
& \vdots \\
& \quad \bar{f}_{k+1}\left(x_{k} ; x_{k-1}, \ldots, x_{2}, t_{1}, t_{2}\right) \\
& \quad=x_{k} \bar{f}_{k}\left(n_{k-1,2} ; n_{k-2,3}, \ldots, n_{2, k-1}, m_{k-1,1}, m_{k-1,2}\right) \\
& \quad+n_{k, 1} \bar{f}_{k}\left(x_{k-1} ; x_{k-2}, \ldots, x_{2}, t_{1}, t_{2}\right)
\end{aligned}
$$

Here $f_{j} \in$ the type $[j]$ for each integer $j \geq 1$ means that in a resolution of the isolated singular point $(0,0)$ of the curve defined by $f_{j}$ there are exactly $j$ exceptional curves each of which has three distinct intersection points with three distinct components among other exceptional curves and the proper transform with normal crossings in the sense of Corollary 1.2.

Theorem 4.2. With Definition 4.1 we have the following properties:

(A) (i) $f_{1} \in$ the type [1] is irreducible in $\mathscr{O}_{0}$ if and only only if $2 \leq a<b$ and $(a, b)=1$.

(ii) $f_{2} \in$ the type [2] is irreducible in $\mathscr{O}_{0}$ if and only if $f_{1}$ is irreducible in $\mathscr{O}_{0}, \bar{f}_{2}\left(m_{11}, m_{12}\right)-a b n_{21}>0$ and $\left(\bar{f}_{2}\left(m_{11}, m_{12}\right), n_{21}\right)=1$.

(iii) For any $k \geq 2, f_{k+1} \in$ the type $[k+1]$ is irreducible in $\mathscr{O}_{0}$ if and only if, for each $j=1, \ldots, k, f_{j} \in$ the type $[j]$ is irreducible in $\mathscr{O}_{0}$,

$$
\begin{aligned}
& \overline{f_{k+1}}\left(n_{k, 2} ; n_{k-1,3}, \ldots, n_{2, k}, m_{k, 1}, m_{k, 2}\right) \\
& \quad-n_{k+1,1} n_{k, 1} \bar{f}_{k}\left(n_{k-1,2} ; n_{k-2,3}, \ldots, n_{2, k-1}, m_{k-1,1}, m_{k-1,2}\right)>0
\end{aligned}
$$

and

$$
\left(\overline{f_{k+1}}\left(n_{k, 2} ; n_{k-1,3}, \ldots, n_{2, k}, m_{k, 1}, m_{k, 2}\right), n_{k+1,1}\right)=1 \text {. }
$$

(B) If $f$ is irreducible in $\mathscr{O}_{0}$ with its multiplicity $n \geq 2$, then $f \sim f_{k+1}$ for some $f_{k+1} \in$ the type $[k+1]$.

(C) The integers $a, b, n_{21}, \ldots, n_{k+1,1} ; \bar{f}_{2}\left(m_{11}, m_{12}\right), \bar{f}_{3}\left(n_{22} ; m_{21}, m_{22}\right)$, $\ldots, \overline{f_{k+1}}\left(n_{k, 2} ; n_{k-1,3}, \ldots, n_{2, k}, m_{k, 1}, m_{k, 2}\right)$ are topologically invariant.

Proof. (A) It is trivial to prove cases (i) and (ii), as we have seen in $\S 3$. To prove one side of $(\mathrm{A})$ by induction index on $k$, let $f_{k+1} \in$ the type $[k+1]$ be irreducible in $\mathscr{O}_{0}$. Then Hensel's lemma and blow-ups imply that $(a, b)=1$. After a finite number $h$ of blow-ups until the total order of the $h$ th exceptional curve $E_{h}=\{v=0\}$ becomes $a b n_{21} \cdots n_{k+1,1}$ in one of the local coordinates $(u, v)$, the defining equation of the total transform may be given topologically by $v^{a b n_{21} \cdots n_{k+1,1}} F_{k}$, where $F_{k}=F_{k-1}^{n_{k+1,1}}+F_{k-2}^{n_{k, 2}} F_{k-3}^{n_{k-1,3}} \cdots F_{1}^{n_{3, k-1}}(u+1)^{n_{2, k}} v^{t}$ with some integer $t \geq 0$ is irreducible in $\mathbb{C}\{u+1, v\}$ and each $F_{j}$ is a proper transform of $f_{j+1}$ for $j=1, \ldots, k$ in $\mathbb{C}\{u+1, v\}$. So $F_{k} \in$ the type $[k]$ in $\mathbb{C}\{u+1, v\}$, because if $F_{k}$ itself does not belong to the type $[k]$, then $E_{h}$ must be tangent to the curve $F_{k}=0$ and so we may assume that $F_{k} \in$ the type $[k]$ in the sense of Corollary 1.2. By induction each $F_{j} \in$ the type [j] is irreducible in $\mathbb{C}\{u+1, v\}$ for $1 \leq j \leq k$. Since the process of blowups and blow-downs preserves the topological classification of total transforms 
with divisors of irreducible plane curve singularities, each $f_{j} \in$ the type $[j]$ is irreducible in $\mathscr{O}_{0}$ for $1 \leq j \leq k$. Now to prove the remaining part of (A), repeat a finite number of blow-ups until we can find $(k-1)$-exceptional curves each of which has three distinct intersection points with three components among other exceptional curves and the proper transform. Then in one of the local coordinates $(u, v)$ the local defining equation of the proper transform may be given topologically as follows:

$$
\left[(u+1)^{n_{k, 1}}+v^{l_{1}}\right]^{n_{k+1,1}}+(u+1)^{n_{k, 2}} v^{l_{2}},
$$

where

$$
\begin{aligned}
l_{1}= & \bar{f}_{k}\left(n_{k-1,2} ; n_{k-2,3}, \ldots, n_{2, k-1}, m_{k-1,1}, m_{k-1,2}\right) \\
& -n_{k-1,1} n_{k, 1} \overline{f_{k-1}}\left(n_{k-2,2} ; n_{k-3,3}, \ldots, n_{2, k-2}, m_{k-2,1}, m_{k-2,2}\right)
\end{aligned}
$$

and

$$
\begin{aligned}
l_{2}= & \bar{f}_{k}\left(n_{k-1,3} ; n_{k-2,4}, \ldots, n_{2, k}, m_{k, 1}, m_{k, 2}\right) \\
& +n_{k, 2} n_{k-1,1} \overline{f_{k-1}}\left(n_{k-2,2} ; n_{k-3,3}, \ldots, n_{2, k-2}, m_{k-2,1}, m_{k-2,2}\right) \\
& -n_{k+1,1}, n_{k, 1} n_{k-1,1} \overline{f_{k-1}}\left(n_{k-2,2} ; n_{k-3,3}, \ldots, n_{2, k-2}, m_{k-2,1}, m_{k-2,2}\right) .
\end{aligned}
$$

Applying the result in the type [2] to the above proper transform, we see that $0<l_{2} n_{k, 1}+l_{1} n_{k, 2}-n_{k, 1} n_{k+1,1} l_{1}$ and $\left(l_{2} n_{k, 1}+l_{1} n_{k, 2}, n_{k+1,1}\right)=1$ imply the desired result. To prove the converse is trivial.

(B) Let $f=z^{n}+a_{2} y^{\alpha_{2}} z^{n-2}+\cdots+a_{n} y^{\alpha_{n}}$ be irreducible in $\mathscr{O}_{0}$, where the $a_{i}=a_{i}(y)$ are units in $\mathscr{O}_{0}$ if they exist, with $a_{1}=0$. Then we are going to prove (B) by induction on the multiplicity $n$ of $f$ at the origin. Let $d=\left(n, \alpha_{n}\right) \geq 2$, as otherwise there is nothing to prove. Put $n=a d$ and $\alpha_{n}=b d$. After a finite number $h$ of blow-ups until the total order of the $h$ th exceptional curve $E_{h}=\{v=0\}$ becomes $a b d$ in one of the local coordinates $(u, v)$, the defining equation of the total transform may be given topologically by

$$
v^{a b d} F=v^{a b d}\left[(u+1)^{d}+c_{2} v^{\gamma_{2}}(u+1)^{d-2}+\cdots+c_{d} v^{\gamma_{d}}\right],
$$

where the $c_{i}(v)$ are units in $\mathbb{C}\{u+1, v\}$ for $2 \leq i \leq d$ if they exist, and the $\gamma_{i}$ are positive integers. Then we have two cases: (i) $d<\gamma_{d}$, (ii) $d>\gamma_{d}$.

Consider the first case (i). By the induction assumption, for some integer $k$

$$
F=F_{k}=F_{k-1}^{n_{k+1,1}}+F_{k-2}^{n_{k, 2}} F_{k-3}^{n_{k-1,3}} \cdots F_{1}^{n_{3, k-1}}(u+1)^{n_{2, k}} v^{p},
$$

where $F_{i} \in$ the type $[i]$ is irreducible in $\mathbb{C}\{u+1, v\}$ for $1 \leq i \leq k-1$ and some $p \geq 0$. Now the problem is how to construct $f_{k+1} \in$ the type $[k+1]$ such that $f \sim f_{k+1}$. By reversing the blow-up process and induction on $k$, let us assume that $f \sim f_{k+1}=f_{k}^{n_{k+1,1}}+f_{k-1}^{n_{k, 2}} f_{k-2}^{n_{k-1,3}} \cdots f_{2}^{n_{3, k-1}} f_{1}^{n_{2, k}} y^{m_{1}} z^{m_{2}}$ for some integers $m_{1}, m_{2}$ if there are such $m_{1}, m_{2}$, because $f_{1}=z^{a}+y^{b}$ and $f_{2}, \ldots, f_{k}$ can be found easily by induction.

Consider the monomial $y^{m_{1}} z^{t}$ in $f_{k+1}$, where $t=m_{2}+a n_{2, k}+a n_{21} n_{3, k-1}+$ $a n_{21} n_{31} n_{4, k-2}+\cdots+a n_{21} n_{31} \cdots n_{k-1,1} n_{k, 2}$. Then after $h$ blow-ups,

$$
\begin{aligned}
a m_{1}+b m_{2}= & a b\left(n_{21} n_{31} \cdots n_{k, 1} n_{k+1,1}-n_{21} n_{31} \cdots n_{k-1,1} n_{k, 2}\right. \\
& \left.-n_{21} n_{31} \cdots n_{k-2,1} n_{k-1,3}-\cdots-n_{21} n_{3, k-1}\right)+p \\
\geq & a b
\end{aligned}
$$


by the degree of $z$ in $f_{k+1}$. By Lemma 3.2, it can be proved. Similarly, we can prove the second case.

(C) Let $g_{k+1} \in \mathscr{O}_{0}$ be irreducible in the type $[k+1]$. Write $g_{k+1}=g_{k}^{s_{k+1,1}}+$ $g_{k-1}^{s_{k, 2}} g_{k-2}^{s_{k-1,3}} \cdots g_{1}^{s_{2, k}} y^{t_{k, 1}} z^{t_{k, 2}}$, where $g_{1}=z^{\alpha}+y^{\beta}, g_{2}=\left(z^{\alpha}+y^{\beta}\right)^{s_{21}}+y^{t_{11}} z^{t_{12}}, \ldots$ are defined by induction on $k$ and the multiplicity of $g_{k+1}$ is $\alpha s_{21} s_{31} \cdots s_{k+1,1}$ at 0 . Since

$$
\begin{gathered}
a n_{21} n_{31} \cdots n_{k+1,1}=\alpha s_{21} \cdots s_{k+1,1}, \quad b n_{21} n_{31} \cdots n_{k+1,1}=\beta s_{21} \cdots s_{k+1,1}, \\
(a, b)=1 \text { and }(\alpha, \beta)=1,
\end{gathered}
$$

it follows that $a=\alpha$ and $b=\beta$. Since the process of blow-ups and blowdowns preserves the topological classification of total transform with divisors of irreducible plane curve singularities, by induction on $k g_{j} \sim f_{j}$ in $\mathscr{O}_{0}$ for $1 \leq j \leq k$. Then by the result of $(\mathrm{A})$, there is nothing to prove.

Using the result in Theorem 4.2, we consider the expression of irreducible plane curve singularities in terms of the parameter $t$ and define some new notation.

Definition 4.3. Let

$$
\begin{aligned}
& l_{2}=\overline{f_{2}}\left(m_{11}, m_{12}\right)-a b n_{21}, \\
& l_{3}=\overline{f_{3}}\left(n_{22} ; m_{21}, m_{22}\right)-n_{31} n_{21} \overline{f_{2}}\left(m_{11}, m_{12}\right), \\
& \vdots \\
& l_{k+1}=\overline{f_{k+1}}\left(n_{k, 2} ; n_{k-1,3}, \ldots, n_{2, k}, m_{k, 1}, m_{k, 2}\right) \\
& \quad-n_{k+1,1} n_{k, 1} \overline{f_{k}}\left(n_{k-1,2} ; n_{k-2,3}, \ldots, n_{2, k-1}, m_{k-1,1}, m_{k-1,2}\right) .
\end{aligned}
$$

Define $L_{j}=l_{j} \prod_{i=j+1}^{k+1} n_{i 1}$ for $j=2, \ldots, k$.

Let $f=z^{n}+a_{2} y^{\alpha_{2}} z^{n-2}+\cdots+a_{n} y^{\alpha_{n}}$ be irreducible in $\mathscr{O}_{0}$, where each $a_{i}=$ $a_{i}(y)$ is a unit in $\mathscr{O}_{0}$ if it exists. Then we get the following (here $\left(\beta_{1}, \ldots, \beta_{k}\right)$ is a greatest common divisor of $\beta_{1}, \ldots, \beta_{k}$ ):

(1) $f \sim f_{1} \in$ the type [1] if and only if $y=t^{n}$ and $z=t^{\alpha_{n}}$, where $2 \leq n<$ $\alpha_{n}$ and $\left(n, \alpha_{n}\right)=1$ with $a=n$ and $b=\alpha_{n}$.

(2) $f \sim f_{2} \in$ the type [2] if and only if $y=t^{n}$ and $z=t^{\alpha_{n}}+t^{\alpha_{n}+l_{2}}$, where $n>\left(n, \alpha_{n}\right)=n_{21}>\left(n, \alpha_{n}, l_{2}\right)=1$ with $n=a n_{21}$ and $\alpha_{n}=b n_{21}$.

(3) $f \sim f_{3} \in$ the type [3] if and only if $y=t^{n}$ and $z=t^{\alpha_{n}}+t^{\alpha_{n}+L_{2}}+t^{\alpha_{n}+L_{2}+l_{3}}$, where $n>\left(n, \alpha_{n}\right)=n_{21} n_{31}>\left(n, \alpha_{n}, L_{2}\right)=n_{31}>\left(n, \alpha_{n}, L_{2}, l_{3}\right)=1$ with $n=a n_{21} n_{31}$ and $\alpha_{n}=b n_{21} n_{31}$.

$(k+1) f \sim f_{k+1} \in$ the type $[k+1]$ if and only if $y=t^{n}$ and $z=t^{\alpha_{n}}+$ $t^{\alpha_{n}+L_{2}}+\cdots+t^{\alpha_{n}+L_{2}+\cdots+L_{k}}+t^{\alpha_{n}+L_{2}+\cdots+L_{k}+l_{k+1}}$, where

$$
\begin{aligned}
n>\left(n, \alpha_{n}\right) & =n_{21} \cdots n_{k+1,1}>\left(n, \alpha_{n}, L_{2}\right) \\
& =n_{31} \cdots n_{k+1,1}>\cdots>\left(n, \alpha_{2}, L_{2}, \ldots, L_{k}\right) \\
& =n_{k+1,1}>\left(n, \alpha_{2}, L_{3}, \ldots, L_{k}, l_{k+1}\right)=1
\end{aligned}
$$

with $n=a n_{21} \cdots n_{k+1,1}$ and $\alpha_{n}=b n_{21} \cdots n_{k+1,1}$. Thus we have proved the following theorem, too. 
Theorem 4.4 (Zariski). Let $f(y, z)=z^{n}+a_{2} y^{\alpha_{2}} z^{n-2}+\cdots+a_{n} y^{\alpha_{n}}$ be irreducible in $\mathscr{O}_{0}$, where each $a_{i}=a_{i}(y)$ is a unit in $\mathscr{O}_{0}$ if it exists, and the $\alpha_{i}$ are positive integers. Assume that the multiplicity of $f$ at $(0,0)$ is $n \geq 2$. Then the curve defined by $f$ at $(0,0)$ can be described topologically by $y=t^{n}$ and $z=t^{\beta_{1}}+t^{\beta_{2}}+\cdots+t^{\beta_{s}}$ with $\beta_{1}=\alpha_{n}$, where $n<\beta_{1}<\beta_{2}<\cdots<\beta_{s}$ and $n>\left(n, \beta_{1}\right)>\cdots>\left(n, \beta_{1}, \ldots, \beta_{s}\right)=1$. If for a given $f$ there is another homeomorphic parametrization defined by $y=t^{m}$ and $z=t^{\eta_{1}}+t^{\eta_{2}}+\cdots+t^{\eta_{b}}$ where $m<\eta_{1}<\eta_{2}<\cdots<\eta_{b}$ and $m>\left(m, \eta_{1}\right)>\cdots>\left(m, \eta_{1}, \ldots, \eta_{b}\right)=1$, then $n=m, s=b$ and $\beta_{i}=\eta_{i}$. Conversely, the curve defined by the parameter $t$ with the same inequality as above must be irreducible at 0 .

Alternate proof. We are going to prove the theorem by induction on the multiplicity of the above $f$ with the coefficient $a_{1}$ zero, without using Theorem 4.2. First consider such an existence of the above parametrization. If $\left(n, \alpha_{n}\right)=1$, the theorem is easy to prove. Let $d=\left(n, \alpha_{n}\right)>1$, where $n=n_{1} d$ and $\alpha_{n}=k_{1} d$. After $m$ iterations of blow-ups until the total order of the $m$ th exceptional curve $E_{m}=\{v=0\}$ becomes $n_{1} k_{1} d$ in one of the local coordinates $(u, v)$, then the defining equation of the total transform of $V=\{f=0\}$ may be given topologically by

$$
v^{n_{1} k_{1} d} h=v^{n_{1} k_{1} d}\left[(u+1)^{d}+c_{2} v^{\gamma_{2}}(u+1)^{d-2}+\cdots+c_{d} v^{\gamma_{d}}\right],
$$

where the $c_{i}$ are units in $\mathbb{C}\{u+1, v\}$ if they exist, and the $\gamma_{i}$ are positive integers. Consider the local defining equation $h$ for the proper transform $V^{(m)}$. Let $r=\gamma_{d}$ for brevity. Then we have three cases:

(i) $d>r$ and $(d, r)=r$, (ii) $d>r$ and $(d, r)<r$, and (iii) $r>d$.

Consider the first case (i). Rewrite

$$
h=A\left[v^{r}+a_{1}(u+1)^{\delta_{1}} v^{r-1}+\cdots+a_{r}(u+1)^{\delta_{r}}\right]
$$

as the Weierstrass polynomial in the variable $v$, where $A$ is a unit, the $a_{i}=$ $a_{i}(u+1)$ are units in $\mathbb{C}\{u+1, v\}$ if they exist for $i=1, \ldots, r$, and the $\delta_{i}$ are positive integers. Note that $d=\delta_{r}$. Since $d=\delta_{r}$ is a multiple of $r$, by eliminating the coefficient $a_{1}$ of $v^{r-1}, h$ can be rewritten as follows:

$$
A\left[w^{r}+b_{2}(u+1)^{m_{2}} w^{r-2}+\cdots+b_{r}(u+1)^{m_{r}}\right],
$$

where $w=v+\left(a_{1} / r\right)(u+1)^{\delta_{1}}$, the $b_{i}$ are units in $\mathbb{C}\{u+1, v\}$ if they exist, and the $m_{i}$ are positive integers.

Note that $\left(r, m_{r}\right)<r$ by Lemma 3.1. So by the induction assumption $h$ itself can be parametrized topologically by $u+1=t^{r}$ and $w=t^{q_{1}}+t^{q_{2}}+\cdots+t^{q_{g}}$, where $r<q_{1}=m_{r}<q_{2}<\cdots<q_{g}$ and $r>\left(r, q_{1}\right)>\cdots>\left(r, q_{1}, \ldots, q_{g}\right)=$ 1. Then $V^{(m)}$ can also be parametrized topologically in the local coordinate $(u, v)$ by $u+1=t^{r}$ and $v=t^{d}+t^{q_{1}}+\cdots+t^{q_{8}}$ with $d=\delta_{r}=r \delta_{1}$ by Hensel's lemma or Theorem 2.2. Since $V^{(m)}$ may be also described homeomorphically by $u+1=t^{r}+t^{r+q_{1}-d}+t^{r+q_{2}-d}+\cdots+t^{r+q_{g}-d}$ and $v=t^{d}$, the curve $V=\{f=0\}$ can be parametrized topologically by $y=t^{n}$ and $z=t^{\alpha_{n}}+t^{\alpha_{n}+r}+t^{\alpha_{n}+r+q_{1}-d}+$ $\cdots+t^{\alpha_{n}+r+a_{8}-d}$. Note that

$$
n<\alpha_{n}<\alpha_{n}+r<\alpha_{n}+r+q_{1}-d<\cdots<\alpha_{n}+r+q_{g}-d
$$

and

$$
\begin{aligned}
& n>\left(n, \alpha_{n}\right)=d>\left(n, \alpha_{n}, \alpha_{n}+r\right)>\left(n, \alpha_{n}, \alpha_{n}+r, \alpha_{n}+r+q_{1}-d\right) \\
& \quad>\cdots>\left(n, \alpha_{n}, \alpha_{n}+r, \ldots, n+\alpha_{n}+q_{g}-d\right)=1 .
\end{aligned}
$$


Thus the case (i) is proved. Similarly the other cases (ii) and (iii) can be proved. Moreover, the uniqueness of such a parametrization as $y=t^{n}$ and $z=$ $t^{\beta_{1}}+\cdots+t^{\beta_{s}}$ for a given $f$ and the converse of the theorem can be easily proved by induction on $s$. Note that there are exactly $s$ exceptional curves, each of which has three distinct intersection points with three components among other exceptional curves and the proper transform in the sense of Corollary 1.2.

\section{REFERENCES}

1. O. Zariski, Studies in equisingularity. I: Equivalent singularities of plane algebroid curves, Amer. J. Math. 87 (1965), 507-536.

2. __ Studies in equisingularity. III: Saturation of local rings and equisingularity, Amer. J. Math. 90 (1968), 961-1023.

3. H. Laufer, Normal two-dimensional singularities, Ann. of Math. Stud., no. 71, Princeton Univ. Press, Princeton, NJ, 1971.

4. T. Apostol, Introduction to analytic number theory, Springer-Verlag, New York, 1976.

Department of Mathematics, College of Natural Sciences, Seoul National UniverSITY, SEOUL 151-742, KOREA 